\title{
NKG2D: A potential therapeutic target in RA?
}

Mounting evidence suggests that the natural killer $(\mathrm{NK})$ cell activating protein, NKG2D, has a pathogenic role in autoimmune diseases. Data from Andersson and colleagues, published in Arthritis \& Rheumatism, indicates that NKG2D could represent a target for therapy in rheumatoid arthritis (RA).

\section{4 ...joint cellularity and} architectural damage observed in histological sections of arthritic joints were greatly reduced $\$ 7$

The investigators found that NKG2D ligands were present in both RA and osteoarthritis human tissues explants and that NKG2D itself was expressed in around $10 \%$ of $\mathrm{CD} 4^{+} \mathrm{T}$ cells from synovial fluid of patients with RA. NKG2D ligand expression and $\mathrm{NKG}_{2} \mathrm{D}^{+} \mathrm{CD} 4^{+} \mathrm{T}$ cells and NK cells were also detected in paws of mice with collagen-induced arthritis (CIA), in contrast to controls.
Next, the authors tested the therapeutic potential of NKG2D blockade in established disease using a nondepleting antibody, CX5. CX5 prevented disease progression compared to isotype control antibody, as indicated by a reduction in paw swelling and clinical score at days 5 and 10 after onset of CIA. Furthermore, joint cellularity and architectural damage observed in histological sections of arthritic joints were greatly reduced.

This paper also offers some mechanistic insight into the pathogenic activity of NKG2D in RA. They found that $\mathrm{CD}^{+} \mathrm{T}$ cell and $\gamma \delta$ T cell numbers in arthritic paws were reduced by over $50 \%$ after NKG2D blockade. Importantly, the proportions of pathogenic IL-17-producing CD $4^{+} \mathrm{T}$ cells and $\gamma \delta$ T cells were increased in paw isolates as opposed to isolates from blood and lymph nodes, and their numbers decreased after CX5 treatment. The protective action of the NKG2D antibody might not only be exerted through regulation of T cells: after receptor engagement, NK cells were found to reduce their expression of NKG2D, with many of the cells becoming NKG2D negative. This was reflected by a decrease in the cytotoxicity of NK cells isolated from CX5 treated mice or from naïve mice subsequently treated in vitro.

The researchers conclude that biologic blockade of NKG2D could combat RA disease progression and joint destruction by reducing the infiltration of inflammatory cells, dampening NK-dependent killing and by inhibiting the production of pathological IL-17. "The most significant findings relate to re-establishing the importance of the involvement of NKG2D bearing cells in arthritis. This provides a rationale for targeted therapy at this receptor," states Percy Sumariwalla, corresponding author of the study.

David Killock

Original article Andersson, A. K. et al. Blockade of NKG2D ameliorates disease in collagen-induced arthritic mice: a potential pathogenic role in chronic inflammatory arthritis. Arthritis Rheum. doi:10.1002/art.30460 